\title{
ARTHRODESIS OF THE HIP BY CENTRAL DISLOCATION AND ILIO-FEMORAL NAILING
}

\author{
A Preliminary Report
}

\author{
Martin Altchek, New York, United States of America
}

This paper describes a method of hip fusion that allows immediate ambulation without external support. It has been successful in twelve out of thirteen operations, the one failure being in a patient with rheumatoid arthritis.

Hitherto, the drawback to hip fusion has been difficulty in securing it without prolonged fixation in a double hip spica. Plaster fixation also stiffens the knee, and if this is not overcome the patient's disability is worsened.

\section{POSITION OF THE HIP}

Central dislocation of the hip combined with ilio-femoral intramedullary nailing is a method of securing bony fusion without external support. The operation is difficult but I believe the patient benefits from being spared four or more months in a hip spica.

The main difficulty is in getting the best possible position for the fused hip. The best position is with sufficient abduction to correct true shortening, and neutral rotation. The amount of flexion is not so important. With more flexion the patient sits a little better; with less he stands a little straighter. The degree of lordosis and mobility of the lumbar spine vary from patient to patient so one cannot be sure of the amount of flexion needed in any one case.

If there is too much abduction the gait will be awkward and there will be increased strain on the knee. If there is too much adduction the gait will be good, providing the foot is in the neutral position, but a shoe raise will be needed. A little adduction is better than too much abduction. Lateral rotation is also better than medial rotation, which produces a very awkward gait, but the neutral position is best.

\section{THE ADVANTAGES OF CENTRAL DISLOCATION}

Central dislocation by itself destroys the axis of rotation of the hip. It blocks all movement except flexion and extension. A surprisingly deep tunnel can be made in the inner wall of the acetabulum in most arthritic hips. The acetabular fossa is usually shallow and the inner wall quite deep. In addition, the pelvic aperture is cut at an angle of $\mathbf{4 5}$ degrees to the long axis of the body. The head and neck of the femur are reamed into a cylinder and placed into the reamed-out acetabulum. It is difficult to dislodge this even without internal fixation. The superior part of the femoral neck and the trochanter form a stable support, like a T-shaped cane for the pelvis to lean on, with the weight transmitted directly to the femur once central dislocation is complete.

Central dislocation by itself is not sufficient to produce fusion unless it is fixed internally or externally. In addition, although central dislocation prevents abduction and adduction, late adduction will occur if fixation is not used. This is because the upper part of the head of the femur and the upper part of the neck is relatively soft bone. The superior part of the acetabular aperture is made of very dense bone. After central dislocation the adductor muscles of the femur are still strong and the abductors are either not functioning or functioning poorly. The constant pull of the adductors through the long femoral lever will cause the upper part of the neck to give way against the sclerotic acetabulum and adduction of the femur will occur.

One patient fell over ten months after operation. The intramedullary nail broke at the acetabulum, and the hip shifted into adduction. This proved that despite new bone formation the hip had not yet fused, although the nail had held it absolutely still as there was no 
radiological evidence of loosening. The upper half of the nail was removed although firmly embedded in the pelvis. There was enough bone formed to prevent loosening of the central dislocation and although the hip gradually shifted into adduction it finally fused, without the patient having to stop walking or needing a plaster.

Central dislocation provides a broad area of bone contact between the femur and the pelvis, necessary to assure fusion. The head and neck of the femur are in close contact with the acetabular aperture and the inner surface of the denuded greater trochanter makes close contact with the denuded superior lateral acetabular lip. The area around the lesser trochanter makes contact with the inner acetabulum.

\section{THE FUNCTION OF THE NAIL}

The time required for solid bony fusion in my opinion is between six months and a year. When plaster is not used, radiographs can be taken to determine the state of fusion. Certainly one would never remove the nail under one year.

The position of the nail is in line with the line of weight bearing and takes less stress than a transversely placed nail. After fusion has been present for many years the pelvis, from the acetabulum to the posterior superior iliac spine, will hypertrophy. This is the area where the nail is placed and is in line with the weight bearing stress.

The nail has maximum purchase in the pelvis and maximum purchase in the femur. Nails placed transversely will often cause fracture of the shaft of the femur at the base of the nail if they do not loosen, but the intramedullary nail does not weaken the femur and has a much better femoral purchase.

The posterior part of the pelvis just lateral to the sacro-iliac joint is fairly thick bone and in almost any patient will hold an 11 millimetre intramedullary nail in its medullary cavity. Sometimes the sacro-iliac joint is so curved that the nail is started in the ilium lying a little posterior to the sacro-iliac joint itself. In most cases, by placing the nail in this portion of the pelvis the femur will be fixed in an acceptable position. In some cases nailing through this area causes too much abduction. One can then place the nail more laterally, through thinner bone, and get a better position of the femur. Alternatively one can place the nail through the thickest part of the ilium, leave the femur in too much abduction, then remove the nail after some callus has formed in a few months, and put the hip in a plaster spica in the corrected position, or reliance may be placed entirely on the central dislocation and a hip spica and leave the ilio-femoral nailing.

In central dislocation of the hip combined with ilio-femoral nailing the amount of abduction must correspond roughly with the abduction of the nail, otherwise when the nail is driven downwards the neck of the femur may be cracked as it is forced to correspond to the position of the nail. For the beginner a wide pelvic aperture will provide a little leeway if the two do not fit exactly. If the neck is fractured reliance must be placed on the nail and, although a hip spica is not needed, the patient must be more careful than if the central dislocation was intact.

If both the central dislocation and the nailing are properly completed, for all practical purposes the hip is fused at that time. If the patient were to fall it is more likely that the femur would break elsewhere rather than at the hip. Therefore, in a patient with good bone, who is reasonably nimble, walking may be undertaken as if the fusion was already solid. Light, athletic patients will often discard their crutches or stick voluntarily about five weeks after operation. As a rule, we advise a stick or crutches for three months after operation. In all cases when the bone is of normal texture there has been no loosening of the nail or the fixation. Two patients with rheumatoid arthritis with bone softening have been operated upon. In one the nail dropped down into the enlarged medullary canal and the fusion failed, the only failure in fifteen cases. The other patient was unable to hold a crutch or a walker and walked without any support three weeks after operation; fusion occurred readily. 
OPERATION

After hip fusion the muscles between the pelvis and the femur have no effect, and for this reason a wide exposure is justified. The supine position is easiest for central dislocation and estimating the correct angle to ream the acetabulum and femur. The lateral position must then be adopted for introduction of the nail. A Y-shaped incision is made. The limbs of the Y go from the anterior superior iliac spine down to the greater trochanter and up to the greater sciatic notch. The stem of the $\mathrm{Y}$ runs from the greater trochanter down the shaft of the femur for about ten inches. The fascia lata is then opened in line with the skin incision. All muscle insertions into the greater trochanter are cut, both in front and at the back. The gluteus maximus muscle is split in line with its fibres back to the greater sciatic notch. Its insertion into the femur may also be cut. Using a wide osteotome, all muscle fibres are cleaned off the capsule by starting at the trochanter and working towards the ilium. About one and a half inches of the ilium above the acetabulum is cleared of muscle attachments. The capsule of the hip is then removed, and the joint dislocated by lateral rotation and flexion.

The hip must be laterally rotated and flexed so that with the knee flexed the foot points to the opposite shoulder. To do this it may be necessary to put firm traction on the dislocated, laterally rotated head while cutting or snapping adhesions at the inner surface of the base of the neck, near and just above the lesser trochanter.

The head and neck of the femur are now reamed out into a cylinder. Charnley's instruments or a modification of them are useful. The head and neck are reamed out at 45 degrees to the long axis of the femur, ignoring anteversion and retroversion. The 45 degrees varus-valgus angle can easily be spotted by observing the shaft of the femur. By ignoring anteversion or retroversion, reaming in the coronal plane of the body is carried out, irrespective of the angle of anteversion. The acetabular aperture is also reamed in the coronal plane, so that when the two are locked together the knee will point forwards. If the knee is bent at a right angle the plane formed between the femur and the tibia will correspond to the sagittal plane of the body, if the patella is straight ahead. Therefore, by reaming the head and neck out at right angles to the plane formed by the femur and tibia with the knee bent, the head and neck are reamed in the coronal plane. The calcar femorale must not be injured and the trephine must be centred over the true centre of the head of the femur. The acetabulum is then reamed out at the corresponding angle but if the patient is lying on the side it is more difficult to determine the coronal plane. Reaming is done with Charnley's progressively larger side-cutting, bluntended reamers. The first reamer has a sharp point and a protective collar to prevent it from going too deep. The remaining reamers are side-cutting and blunt-ended to prevent damage to the pelvic contents. The outer and upper edge of the acetabulum is then denuded of cartilage. The remainder of the acetabulum which has not been touched should be fish-tailed if time permits. The inner lip of the greater trochanter and its upper edge should be denuded of all soft tissue and cortical bone as the beginning of union in my series occurs between the upper outer lip of the acetabulum and the greater trochanter (Figs. 1 and 2).

Central dislocation is then tested. In many osteoarthritic hips there may be great difficulty in redislocating the hip if the tunnel is deep. If the position is not right it can be changed by widening the acetabular aperture with the largest reamer or redirecting the reamer to allow more varus or valgus. Rotation is most important. This can be judged best by bending the knee to a right angle when the degree of rotation becomes apparent.

Preparation for ilio-femoral nailing is then made. Schneider's bent, self-broaching hip fusion pin, with the drill guide, and a quarter of an inch drill are used. The patient is placed on the side. A second incision is made over the posterior superior iliac spine. About five inches of the posterior ilium is denuded, inside and out, so that the thickest part can be felt through which the nail has to be driven. A point is chosen near the posterior superior iliac spine, deep enough so that the nail will be in bone all the way. 
The acetabular site for the nail is now selected. With the hip reduced, a point in the roof of the acetabulum is chosen where the nail should emerge to go down the medullary canal of the femur and a mark is made. The two points, in the upper ilium and in the acetabulum, are now connected with the drill guide, with the hip dislocated. The drill is placed in the opening of the guide and it points in the direction in which the femur will be placed. With a finger inside the pelvis through the greater sciatic notch, the inner surface of the pelvis can be felt and the nail properly guided through the thickest part. The pelvis is then drilled from

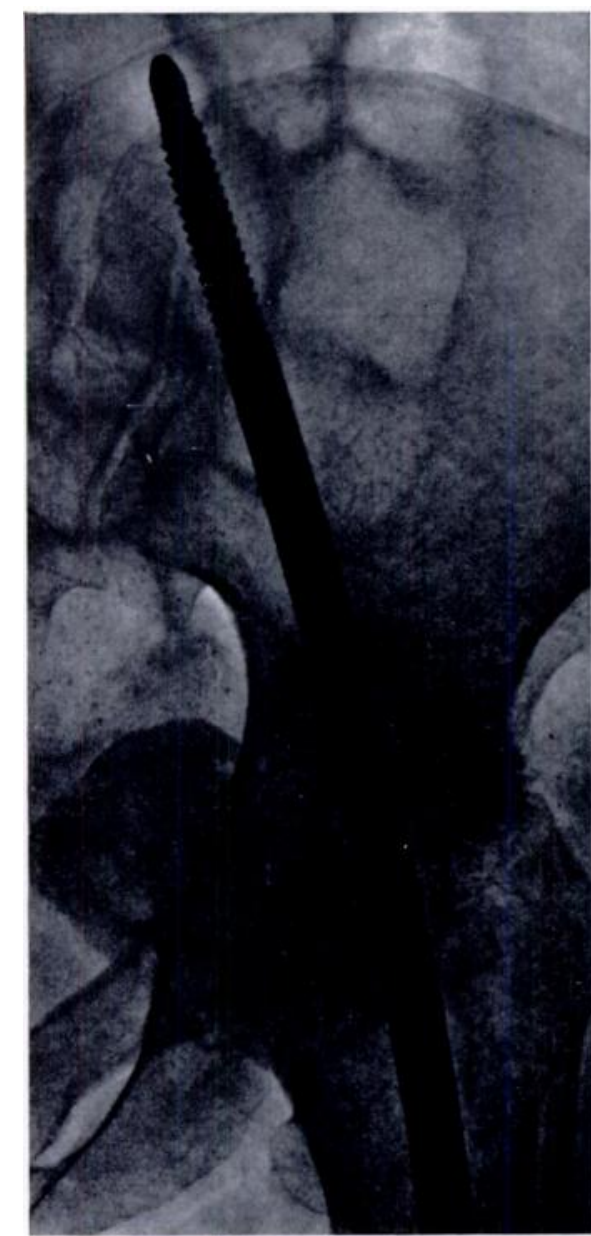

FIG. 1

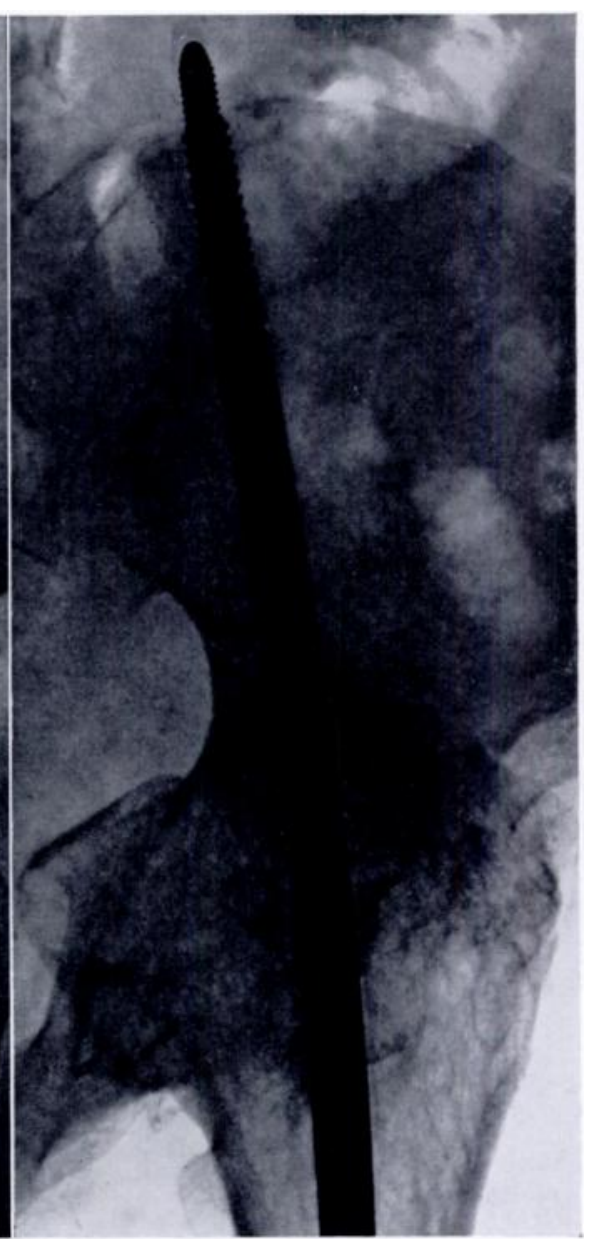

FIG. 2

Figure 1-The left hip of a woman of 41 with osteoarthritis one month after operation showing the position of the nail and the snug fit of the greater trochanter against the outer acetabular margin. Figure 2-The same patient two and a half years after operation showing fusion of the hip, most marked at the acetabulo-trochanteric junction.

below upwards, using a quarter-inch drill; a finger in the greater sciatic notch makes sure that the drill does not go into the pelvis or out of the ilium above the acetabular roof. The pelvis is drilled for about four inches and the guide removed. The nail is then inserted. It is driven from the acetabulum upwards, to come out near the posterior superior iliac spine, and once started for an inch or more its position will not change. It is best to have the ilium grasped between forefinger and thumb to make sure that the nail is not going astray, and the nail is driven upwards until its lower end is flush with the acetabulum. 
In placing the nail, the curve of the nail may be used to reduce flexion or abduction. By placing the convex surface of the nail anteriorly, the curve of the nail serves to reduce flexion; by placing the convex surface laterally, the curve of the nail tends to reduce abduction. Usually a mid position is used.

Once the lower end of the nail is flush with the acetabulum the femur is reduced into the acetabular aperture, after a hole has been made in the femur in the upper surface of the base of its neck in order to give the nail a start. With the hip reduced, the nail is then driven downwards into the femoral shaft. Usually it is easier than it sounds to get the nail down the meduallary canal. Flexion and extension of the femur may be changed before the nail enters the femur. If abduction or adduction needs to be corrected the direction of the acetabular aperture may be enlarged before driving the nail into the femur. It is important that the hip is in neutral rotation. When the nail is being driven downwards the knee should be bent to a right angle so as to make any femoral rotation obvious. Once the nail is down fixation is solid. Usually the head and neck of the femur fit so snugly into the acetabular aperture, with the greater and lesser trochanters against the pelvis, that there is little room to add more bone. The gluteal muscles and fascia are sutured together in one layer and the subcutaneous layer and the skin are closed. Vacuum suction tubes are placed inside the pelvis through the greater sciatic notch and around the site of fusion.

Walking is allowed as soon as the patient feels like it, usually a week after operation. The absolute fixation reduces pain and full weight bearing is allowed. Crutches or a walker are used for three months, but lighter, more agile patients may discard support as soon as they are able to balance.

\section{RESULTS}

Fifteen cases have been operated on so far. Twelve have fused. The time since operation of two others is too short for assessment. None has needed external fixation. One failure occurred in a patient with rheumatoid arthritis and osteoporotic bone. One patient whose hip was fixed in too much abduction conveniently fell about three weeks after operation, sustaining a spiral fracture of the femur in its middle third. The hip fusion was not disturbed. A hip spica was applied for eight weeks with the femur in a corrected position, overcoming the abduction. The femur united and the final result was excellent. In one patient, a man of seventy, six feet one inch tall, and 270 pounds in weight, the neck of the femur was fractured when the nail was driven downwards. This happened because the nail fixed the femur in more adduction than the central dislocation allowed and forcefully adducted the femur as the nail was driven downwards. It could have been prevented by making the acetabular aperture in more valgus. Nevertheless, he was allowed up walking in a walker, and the hip appears to be fusing after four months, held by the nail alone.

\section{SUMMARY}

1. Central dislocation of the hip combined with ilio-femoral intramedullary nailing is a technically difficult operation, but one which, if well done, provides a high percentage of hip fusion without external fixation.

2. Fifteen hips have been operated upon. Twelve have fused; two cannot yet be assessed as the time since operation is too short; and one failed, presumably due to osteoporosis resulting from rheumatoid arthritis. 